\title{
开式洜控非对称缸系统一阶轨迹灵敏度分析 ${ }^{*}$
}

\author{
姚 静 $1,2,3$ 蒋东廷 ${ }^{3}$ 张 伟 ${ }^{3}$ 董兆胜 ${ }^{3}$
}

(1. 燕山大学河北省重型机械流体动力传输与控制实验室 秦皇岛 066004;

2. 先进锻压成形技术与科学教育部重点实验室(燕山大学) 秦皇岛 066004;

3. 燕山大学机械工程学院 秦皇岛 066004)

\begin{abstract}
摘要: 原控技术在工程机械、重型机械等领域应用广泛，但目前对其输出特性的分析较少。为了探究洜控系统关键参数对 系统输出特性的影响, 基于开式洜控非对称缸系统状态方程建立系统一阶轨迹灵敏度模型, 并求出各参数的灵敏度函数曲 线。提出了峰值灵敏度、均值灵敏度两个衡量指标, 分析各参数变化对位移输出特性影响程度的大小。基于 $0.6 \mathrm{MN}$ 开式

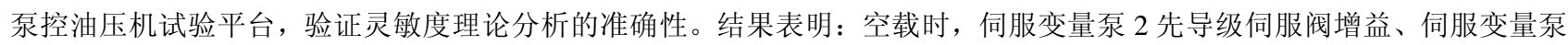
2 和伺服变量葲 1 的先导级伺服阀时间常数影响较大; 加载时, 各参数两项灵敏度指标数值相近, 其中伺服变量百 2 的先 导级伺服阀的增益、时间常数和油缸面积以及流量增益、系统比例增益影响较大。分析结果可为泵控非对称缸系统性能优 化提供理论依据。
\end{abstract}

关键词: 原控系统; 非对称缸; 灵敏度; 状态方程

中图分类号: $\mathrm{TH} 137$

\section{First-order Trajectory Sensitivity Analysis of Open Circuit Pump- controlled Asymmetric Cylinder Systems}

\author{
YAO Jing $^{1,2,3}$ JIANG Dongting ${ }^{3} \quad$ ZHANG Wei $^{3}$ DONG Zhaosheng ${ }^{3}$
}

(1. Hebei Province Key Laboratory of Heavy Machinery Fluid Power Transmission and Control, Yanshan University, Qinhuangdao 066004;

2. Key Laboratory of Advanced Forging \& Stamping Technology and Science of Ministry of Education, Yanshan University, Qinhuangdao 066004;

3. School of Mechanical Engineering, Yanshan University, Qinhuangdao 066004)

\begin{abstract}
The pump-controlled technology has wide application in engineering machinery, heavy machinery and other fields. However, there are few analyses on its output characteristics at present. To explore the effect of the critical parameters of the pump control system on the output characteristics of the system, based on the state equations of open circuit pump- controlled asymmetric cylinder systems, the first-order trajectory sensitivity equations are derived and the sensitivity function curves of each parameter are obtained. Two measures of peak sensitivity and mean sensitivity are proposed to analyze the influence degree of each parameter variation on the displacement output characteristics. Based on 0.6MN pump-controlled hydraulic forging test bench, the accuracy of sensitivity theory analysis is verified. Experimental results indicate that in no-load time, servo variable pump 2 pilot servo valve gain, servo variable pump 2 and servo variable pump 1 pilot stage servo valve time constant has great influence. In load, that value of the sensitivity indexes of two parameter of each parameter is similar, in which the gain of pilot stage servo valve of servo variable pump 2, time constant and oil cylinder area and flow gain and system ratio gain are greatly affected. All the research above can provide theoretical basis for pump-controlled asymmetric cylinder systems performance optimization.
\end{abstract}

Key words: pump-controlled system; asymmetric cylinder; sensitivity analysis; state equations

* 国家自然科学基金(51575471)、河北省教育厅高等学校科技计划重点项 目(ZD2017077)和流体动力与机电系统国家重点实验室2017年开放基金 (GZKF-201716)资助项目。20180310 收到初稿, 20181012 收到修改稿 


\section{0 前言}

随着人们对能源短缺担忧的日益加剧, 液压传 动系统节能性研究受到了广泛的关注。为了降低液 压系统的能耗, 最直接的方法就是采用原控技术, 原控技术与传统的阀控技术相比具有结构简单、传 动效率高、无节流损失和溢流损失等优点, 一直备 受学者和工程技术人员的关注 ${ }^{[1-2]}$ 。

近年来国内外学者提出了原控对称缸系统、㰮 控非对称缸系统、泵控差动缸系统等多种洜控系 统 ${ }^{[3]}$, 并且在工程机械 ${ }^{[4-5]}$ 、锻压设备 ${ }^{[6]}$ 、船用设备 ${ }^{[7]}$ 等领域得到了广泛的应用。但洜控系统仍有响应速 度慢、严重的非线性和控制精度低等问题, 针对上 述问题, 国内外学者提出了多种控制方法, 如自适 应控制方法 ${ }^{[8-9]}$ 、非线性控制方法 ${ }^{[10]} 、$ 混合控制方法 ${ }^{[11]}$ 、 基于泵控对称缸系统的简单自适应控制方法 ${ }^{[12]}$ 、基 于原控差动缸系统的广义模糊 PID 控制方法 ${ }^{[13]}$, 取 得了较好的控制效果。虽然采用先进的控制理论在 一定程度上能改善泵控系统的动静态特性, 但是, 探索百控系统各个关键参数对系统输出特性的影响 程度, 得到决定性的影响参数, 为控制器设计提供 参考, 必将大幅度提高泵控系统的动静态特性。又 鉴于非对称缸结构简单、占用空间少、承载能力大、 市场应用广泛等特点, 因此, 本文将针对百控非对 称缸系统进行灵敏度分析。灵敏度分析方法可以定 量分析各参数变化对系统动态特性影响程度的大 小, 并且在电力系统 ${ }^{[14]}$ 、水利系统 ${ }^{[15]}$ 、机械系统的 设计及分析 ${ }^{[16-17]}$ 等领域取得了广泛的应用。但灵敏 度理论在液压领域的应用相对较少。近年来, 国内 外学者将灵敏度分析方法用于阀控缸系统分析, 得 到了阀控缸系统主要参数对系统动静态特性的影响 程度后又提出不同灵敏度分析方法, 并对比分析各 灵敏度分析方法的异同点 ${ }^{[18]}$, 为液压系统建模分析 及非线性控制 ${ }^{[19-20]}$ 、液压元件的设计 ${ }^{[21]}$ 等方面提供 理论基础。

本文以泵控非对称缸系统为研究对象, 采用一 阶轨迹灵敏度分析方法, 建立系统的灵敏度方程, 分析主要参数在位移阶跃响应激励下对位移的灵敏 度, 为泵控非对称缸系统性能优化提供理论依据。

\section{1 开式洜控非对称缸系统模型}

\section{1 系统原理}

开式泵控非对称缸系统原理如图 1 所示, 系统 主要由三联洜、数据采集控制系统、液压缸、传感
器等元件组成。

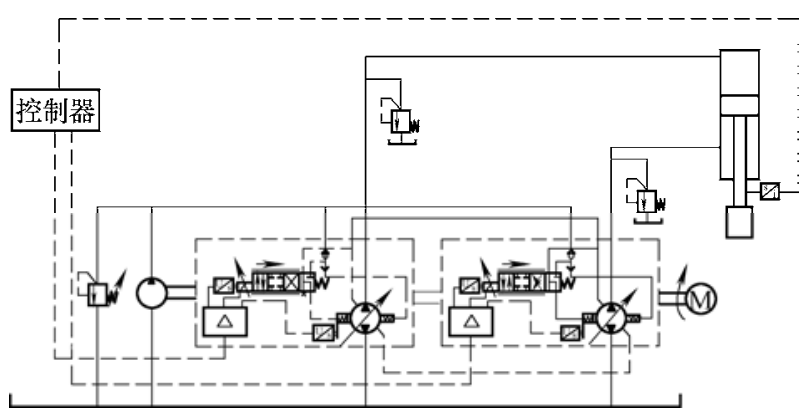

图 1 开式泵控非对称缸系统原理

系统采用负载独立容积控制, 即两个变量洜单 独对非对称缸的两腔容积进行控制, 它不仅解决了 非对称液压缸两腔流量不对称问题, 提高了系统的 控制自由度, 还能进行超越负载工况下的能量回收, 同时降低了装机功率和系统冷却功率。省去低压补 油的大流量补油泵, 进而提高了能量利用效率 ${ }^{[22]}$ 。

\section{2 开式洜控非对称缸数学模型}

变量泵作为泵控系统的核心元件, 其参数对系 统控制性能的影响较大。本文选取 MOOG 公司的比 例变量径向柱塞泵(RKP 变量泵)作为动力源。该洜 具有动态响应快、噪声低、压力波动小等优点。

忽略管道和阀腔内的压力损失, 假定液体是不 可压缩的, 并认为变量愿的吸油口压力为零, 则变 量百的流量经拉氏变换可表示为 ${ }^{[23]}$

$$
Q_{p}=\frac{K_{p q} K_{s} K_{a}\left(U_{\text {in }}-K_{x} X_{s}\right)}{\left(T_{s} s+1\right) A_{s} s}-C_{t p} P
$$

式中, $K_{p q}$ 为变量百的流量增益 $\left(\mathrm{m}^{2} / \mathrm{s}\right), K_{s}$ 为伺服阀增 益 $(\mathrm{A} / \mathrm{V}), T_{s}$ 为伺服阀时间常数(s), $K_{a}$ 为放大器增益 $(\mathrm{A} / \mathrm{V}), U_{\text {in }}$ 为伺服阀指令电压信号 $(\mathrm{V}), U_{f}$ 为伺服阀反 馈电压信号 $(\mathrm{V}), K_{x}$ 冲程环位置增益 $(\mathrm{V} / \mathrm{m}), X_{s}$ 冲程环 偏心距 $(\mathrm{m}), P$ 为变量泵口的压力 $(\mathrm{Pa}), C_{t p}$ 为变量泵的 外泄漏系数 $\left(\mathrm{m}^{3} / \mathrm{s} \cdot \mathrm{Pa}\right), A_{s}$ 为伺服油缸面积 $\left(\mathrm{m}^{2}\right)$ 。

\section{3 状态方程}

一般系统的状态方程可以表示为

$$
\dot{\boldsymbol{x}}=\boldsymbol{f}(\boldsymbol{x}, \boldsymbol{u}, \boldsymbol{\alpha}, t)
$$

式中, $\boldsymbol{x}$ 为 $n$ 维状态矢量, $\boldsymbol{u}$ 为与 $\boldsymbol{\alpha}$ 无关的 $r$ 维输 入, $\boldsymbol{a}$ 为 $p$ 维参数, $t$ 为时间。

开式洜控非对称缸系统最高阶次为 8 阶, 这里 选取 8 个状态变量、 1 个输入和 21 个参数项, 则式 (2)的各矢量可表示为

$$
\begin{aligned}
\boldsymbol{x}= & \left(x_{1}, x_{2}, x_{3}, x_{4}, x_{5}, x_{6}, x_{7}, x_{8}\right)^{\mathrm{T}} \\
\boldsymbol{u}= & \left(u_{1}\right)^{\mathrm{T}} \\
\boldsymbol{\alpha}= & \left(\alpha_{1}, \alpha_{2}, \alpha_{3}, \alpha_{4}, \alpha_{5}, \alpha_{6}, \alpha_{7}, \alpha_{8}, \alpha_{9}, \alpha_{10}, \alpha_{11}, \alpha_{12},\right. \\
& \left.\alpha_{13}, \alpha_{14}, \alpha_{15}, \alpha_{16}, \alpha_{17}, \alpha_{18}, \alpha_{19}, \alpha_{20}, \alpha_{21}\right)^{\mathrm{T}}
\end{aligned}
$$


式中, 状态矢量 $\boldsymbol{x}$ 中的状态变量为

$$
\begin{aligned}
& x_{1}=Q_{s 1}, \quad x_{2}=Q_{s 2}, \quad x_{3}=X_{s 1}, \quad x_{4}=X_{s 2}, \\
& x_{5}=P_{1}, \quad x_{6}=P_{2}, \quad x_{7}=y, \quad x_{8}=\dot{y}
\end{aligned}
$$

输入矢量 $\boldsymbol{u}$ 中的输入为

$$
u_{1}=Y_{\text {in }}
$$

参数矢量 $\boldsymbol{\alpha}$ 中的参数为

$$
\begin{array}{llll}
\alpha_{1}=K_{s 1}, & \alpha_{2}=K_{s 2}, & \alpha_{3}=T_{s 1}, & \alpha_{4}=T_{s 2}, \\
\alpha_{5}=A_{s 1}, & \alpha_{6}=A_{s 2}, & \alpha_{7}=K_{p}, & \alpha_{8}=K_{q p 1}, \\
\alpha_{9}=K_{q p 2}, & \alpha_{10}=l_{0}, & \alpha_{11}=C_{t p 1}, & \alpha_{12}=C_{t p 2}, \\
\alpha_{13}=A_{1}, & \alpha_{14}=A_{2}, & \alpha_{15}=C_{e c 1}, & \alpha_{15}=C_{e c 2}, \\
\alpha_{16}=C_{e c 2}, & \alpha_{17}=B_{e}, & \alpha_{18}=m_{t}, & \alpha_{19}=B_{p}, \\
\alpha_{20}=F_{L}, & \alpha_{21}=K &
\end{array}
$$

即式(2)可整理为

$$
\begin{gathered}
\dot{x}_{1}=\frac{K_{a 1} K_{s 1}\left[K_{p}\left(Y_{\mathrm{in}}-x_{7} K_{w}\right)-x_{3} K_{x}\right]-x_{1}}{T_{\mathrm{s} 1}} \\
\dot{x}_{2}=\frac{K_{a 2} K_{s 2}\left[K_{p} K_{b}\left(Y_{\mathrm{in}}-x_{7} K_{w}\right)-x_{4} K_{x}\right]-x_{2}}{T_{\mathrm{s} 2}} \\
\dot{x}_{3}=\frac{x_{1}}{A_{s 1}} \\
\dot{x}_{5}=\frac{\dot{x}_{4}=\frac{x_{2}}{A_{\mathrm{s} 2}}}{B_{e}\left[x_{3} K_{q p 1}-\left(C_{t p 1}+C_{e c 1}\right) x_{5}-A_{1} x_{8}\right]} \\
A_{1}\left(l_{0}+x_{7}\right) \\
\dot{x}_{6}=\frac{-B_{e}\left[x_{4} K_{q p 2}+\left(C_{t p 2}+C_{e c 2}\right) x_{6}-A_{2} x_{8}\right]}{A_{2}\left(L-l_{0}-x_{7}\right)} \\
\dot{x}_{7}=x_{8} \quad A_{8}=\frac{A_{1} x_{5}-A_{2} x_{6}-B_{p} x_{8}-K x_{7}-F_{L}}{m_{t}}
\end{gathered}
$$

\section{2 一阶灵敏度求解}

\section{1 一阶轨迹灵敏度方程}

目前, 灵敏度有众多模型, 其中一阶轨迹灵敏 度模型适用于输入因素个数少、结构不复杂、灵敏 度微分方程较易推导的系统或模型, 并且相较于其 他高阶灵敏度模型不仅具有计算简便等优点而且能 够保证较高的精度。对于百控非对称缸系统, 其系 统结构简单, 仅有一个输入参数 $y$, 故选用一阶灵敏 度模型对开式百控非对称缸系统进行灵敏度分析。

式(2)的解可以表示为

$$
\varphi_{n}(t)=\boldsymbol{x}(t, \boldsymbol{\alpha})_{n}, n=1,2, \cdots, 8
$$

状态矢量 $\boldsymbol{x}$ 对参数 $\boldsymbol{\alpha}$ 的灵敏度函数定义为

$$
\lambda_{n}^{i}=\left(\frac{\partial \boldsymbol{x}}{\partial a_{i}}\right)_{n}, \quad i=1,2, \cdots, 21
$$

其初始条件为

$$
\lambda_{0}^{i}=\left(\frac{\partial x_{0}}{\partial a_{i}}\right)_{n}, \quad i=1,2, \cdots, 21
$$

在 $\boldsymbol{u}$ 与 $\boldsymbol{\alpha}$ 相互独立的情况下，式(4)在等式两边 同时对参数矢量 $\boldsymbol{\alpha}$ 求偏导数得

$$
\dot{\lambda}_{n}^{i}=\left(\frac{\partial \boldsymbol{f}}{\partial \boldsymbol{x}}\right)_{n} \lambda_{n}^{i}+\left(\frac{\partial \boldsymbol{f}}{\partial \alpha_{i}}\right)_{n}, \quad i=1,2, \cdots, 21
$$

式(6)为一阶轨迹灵敏度方程，式中 $(\partial \boldsymbol{f} / \partial \boldsymbol{x})_{n}$ 为灵敏 度方程系数项， $\left(\partial \boldsymbol{f} / \partial \alpha_{i}\right)_{n}$ 为灵敏度方程自由项。

\section{2 系数矩阵及自由项矩阵}

根据灵敏度方程(6), 求式(2)中函数 $\boldsymbol{f}(\boldsymbol{x}, \boldsymbol{u}, \boldsymbol{\alpha}, \mathrm{t})$ 对状态矢量 $\boldsymbol{x}$ 的偏导数, 可得如下雅可比矩阵

$$
\frac{\partial f}{\partial x_{n}}=\left(\begin{array}{lllllc}
a_{1,1} & a_{1,2} & \cdots & a_{1, n} & \cdots & a_{1,8} \\
a_{2,1} & a_{2,2} & & & & \vdots \\
\vdots & & \ddots & & & \vdots \\
a_{n, 1} & & & a_{n, n} & & \vdots \\
\vdots & & & & \ddots & \vdots \\
a_{8,1} & \cdots & \cdots & \cdots & \cdots & a_{8,8}
\end{array}\right)
$$

式(7)中各元素可具体表示为

$$
\begin{aligned}
& \frac{\partial f}{\partial x_{1}}=\left(-\frac{1}{T_{s 1}}, 0, \frac{1}{A_{s 1}}, 0,0,0,0,0\right)^{\mathrm{T}} \\
& \frac{\partial f}{\partial x_{2}}=\left(0,-\frac{1}{T_{s 2}}, 0, \frac{1}{A_{s 2}}, 0,0,0,0\right)^{\mathrm{T}} \\
& \frac{\partial f}{\partial x_{3}}=\left(-\frac{K_{a 1} K_{s 1} K_{x}}{T_{s 1}}, 0,0,0, \frac{B_{e} K_{q p 1}}{A_{1}\left(l_{0}+x_{7}\right)}, 0,0,0\right)^{\mathrm{T}} \\
& \frac{\partial f}{\partial x_{4}}=\left(0,-\frac{K_{a 2} K_{s 2} K_{x}}{T_{s 2}}, 0,0,0,-\frac{B_{e} K_{q p 2}}{A_{2}\left(L-l_{0}-x_{7}\right)}, 0,0\right)^{\mathrm{T}} \\
& \frac{\partial f}{\partial x_{5}}=\left(0,0,0,0,-\frac{B_{e}\left(C_{t p 1}+C_{e c 1}\right)}{A_{1}\left(l_{0}+x_{7}\right)}, 0,0, \frac{A_{1}}{m_{t}}\right)^{\mathrm{T}} \\
& \frac{\partial f}{\partial x_{6}}=\left(0,0,0,0,0,-\frac{B_{e}\left(C_{t p 2}+C_{e c 2}\right)}{A_{2}\left(L-l_{0}-x_{7}\right)}, 0,-\frac{A_{2}}{m_{t}}\right)^{\mathrm{T}} \\
& \frac{\partial f}{\partial x_{7}}=\left(-\frac{K_{p} K_{s 1} K_{w} K_{a 1}}{T_{s 1}},-\frac{K_{p} K_{s 2} K_{w} K_{a 2} K_{b}}{T_{s 2}}, 0,0,\right. \\
& -\frac{A_{1} B_{e}\left[x_{3} K_{q p 1}-\left(C_{t p 1}+C_{e c 1}\right) x_{5}-A_{1} x_{8}\right]}{\left[A_{1}\left(l_{0}+x_{7}\right)\right]^{2}}, \\
& \left.-\frac{A_{2} B_{e}\left[x_{4} K_{q p 2}+\left(C_{t p 2}+C_{e c 2}\right) x_{6}-A_{2} x_{8}\right]}{\left[A_{2}\left(L-l_{0}-x_{7}\right)\right]^{2}}, 0,-\frac{K}{m_{t}}\right)^{\mathrm{T}}
\end{aligned}
$$




$$
\frac{\partial f}{\partial x_{8}}=\left(0,0,0,0,-\frac{A_{1} B_{e}}{A_{1}\left(l_{0}+x_{7}\right)}, \frac{A_{2} B_{e}}{A_{2}\left(L-l_{0}-x_{7}\right)}, 1,-\frac{B_{p}}{m_{t}}\right)^{\mathrm{T}}
$$

上述求得 8 阶方阵, 为式(6)灵敏度函数的系数 项矩阵。

求式(2)中函数 $\boldsymbol{f}(\boldsymbol{x}, \boldsymbol{u}, \boldsymbol{\alpha}, \mathrm{t})$ 对参数矢量 $\boldsymbol{\alpha}$ 的偏导 数, 得出如下矩阵

$$
\frac{\partial f}{\partial a_{i}}=\left(\begin{array}{cccccc}
b_{1,1} & b_{1,2} & \cdots & b_{1, \mathrm{i}} & \cdots & b_{1,21} \\
b_{2,1} & b_{2,2} & & & & \vdots \\
\vdots & & \ddots & & & \vdots \\
b_{\mathrm{n}, 1} & & & b_{n, i} & & \vdots \\
\vdots & & & & \ddots & \vdots \\
b_{8,1} & \cdots & \cdots & \cdots & \cdots & b_{8,21}
\end{array}\right)
$$

式(8) 中各元素可具体表示为

$$
\begin{aligned}
& \frac{\partial f}{\partial \alpha_{1}}=\left(\frac{K_{a 1}\left[\left(Y_{\text {in }}-x_{7} K_{w}\right) K_{p}-x_{3} K_{x}\right]}{T_{s 1}}, 0,0,0,0,0,0,0\right)^{\mathrm{T}} \\
& \frac{\partial f}{\partial \alpha_{1}}=\left(\frac{K_{a 1}\left[\left(Y_{\text {in }}-x_{7} K_{w}\right) K_{p}-x_{3} K_{x}\right]}{T_{s 1}}, 0,0,0,0,0,0,0\right)^{\mathrm{T}} \\
& \frac{\partial f}{\partial \alpha_{2}}=\left(0, \frac{K_{a 2}\left[\left(Y_{\text {in }}-x_{7} K_{w}\right) K_{p} K_{b}-x_{4} K_{x}\right]}{T_{s 2}}, 0,0,0,0,0,0\right)^{\mathrm{T}}
\end{aligned}
$$

$$
\frac{\partial f}{\partial \alpha_{3}}=\left(-\frac{K_{a 1} K_{s 1}\left[\left(Y_{\mathrm{in}}-x_{7} K_{w}\right) K_{p}-x_{3} K_{x}\right]-x_{1}}{T_{s 1}^{2}},\right.
$$

$$
0,0,0,0,0,0,0)^{\mathrm{T}}
$$

$$
\frac{\partial f}{\partial \alpha_{4}}=\left(0,-\frac{K_{a 2} K_{s 2}\left[\left(Y_{\text {in }}-x_{7} K_{w}\right) K_{p} K_{b}-x_{4} K_{x}\right]-x_{2}}{T_{\text {s2 }}{ }^{2}},\right.
$$

$$
0,0,0,0,0,0)^{\mathrm{T}}
$$

$$
\begin{aligned}
& \frac{\partial f}{\partial \alpha_{5}}=\left(0,0,-\frac{x_{1}}{A_{s 1}^{2}}, 0,0,0,0,0\right)^{\mathrm{T}} \\
& \frac{\partial f}{\partial \alpha_{6}}=\left(0,0,0,-\frac{x_{2}}{A_{s 2}^{2}}, 0,0,0,0\right)^{\mathrm{T}}
\end{aligned}
$$$$
\frac{\partial f}{\partial \alpha_{7}}=\left(\frac{K_{a 1} K_{s 1}\left(Y_{\text {in }}-x_{7} K_{w}\right)}{T_{s 1}}, \frac{K_{a 2} K_{s 2} K_{b}\left(Y_{\text {in }}-x_{7} K_{w}\right)}{T_{s 2}},\right.
$$

$$
0,0,0,0,0,0)^{\mathrm{T}}
$$

$$
\frac{\partial f}{\partial \alpha_{8}}=\left(0,0,0,0, \frac{B_{e} x_{3}}{A_{1}\left(l_{0}+x_{7}\right)}, 0,0,0\right)^{\mathrm{T}}
$$

$$
\frac{\partial f}{\partial \alpha_{9}}=\left(0,0,0,0,0,-\frac{B_{e} x_{4}}{A_{2}\left(L-l_{0}-x_{7}\right)}, 0,0\right)^{\mathrm{T}}
$$

$$
\begin{aligned}
& \frac{\partial f}{\partial \alpha_{10}}=\left(0,0,0,0,-\frac{A_{1} B_{e}\left[x_{3} K_{q p 1}-\left(C_{t p 1}+C_{e c 1}\right) x_{5}-A_{1} x_{8}\right]}{\left[A_{1}\left(l_{0}+x_{7}\right)\right]^{2}}\right. \\
& \left.-\frac{A_{2} B_{e}\left[x_{4} K_{q p 2}+\left(C_{t p 2}+C_{e c 2}\right) x_{6}-A_{2} x_{8}\right]}{\left[A_{2}\left(L-l_{0}-x_{7}\right)\right]^{2}}, 0,0\right)^{\mathrm{T}} \\
& \frac{\partial f}{\partial \alpha_{11}}=\left(0,0,0,0,-\frac{B_{e} x_{5}}{A_{1}\left(l_{0}+x_{7}\right)}, 0,0,0\right)^{\mathrm{T}} \\
& \frac{\partial f}{\partial \alpha_{12}}=\left(0,0,0,0,0,-\frac{B_{e} x_{6}}{A_{2}\left(L-l_{0}-x_{7}\right)}, 0,0\right)^{\mathrm{T}} \\
& \frac{\partial f}{\partial \alpha_{13}}=\left(0,0,0,0, \frac{-B_{e}\left(l_{0}+x_{7}\right)\left[x_{3} K_{q p 1}-\left(C_{t p 1}+C_{e c 1}\right) x_{5}\right]}{\left[A_{1}\left(l_{0}+x_{7}\right)\right]^{2}},\right. \\
& \left.0,0, \frac{x_{5}}{m_{t}}\right)^{\mathrm{T}} \\
& \frac{\partial f}{\partial \alpha_{14}}=(0,0,0,0,0 \text {, } \\
& \frac{B_{e}\left(L-l_{0}-x_{7}\right)\left[x_{3} K_{q p 1}-\left(C_{t p 1}+C_{e c 1}\right) x_{5}-A_{1} x_{8}\right]}{\left[A_{2}\left(L-l_{0}-x_{7}\right)\right]^{2}}, \\
& \left.0, \frac{x_{5}}{m_{t}}\right)^{\mathrm{T}} \\
& \frac{\partial f}{\partial \alpha_{15}}=\left(0,0,0,0,-\frac{B_{e} x_{5}}{A_{1}\left(l_{0}+x_{7}\right)}, 0,0,0\right)^{\mathrm{T}} \\
& \frac{\partial f}{\partial \alpha_{16}}=\left(0,0,0,0,0,-\frac{B_{e} x_{6}}{A_{2}\left(L-l_{0}-x_{7}\right)}, 0,0\right)^{\mathrm{T}} \\
& \frac{\partial f}{\partial \alpha_{17}}=\left(0,0,0,0, \frac{x_{3} K_{q p 1}-\left(C_{t p 1}+C_{e c 1}\right) x_{5}-A_{1} x_{8}}{A_{1}\left(l_{0}+x_{7}\right)},\right. \\
& \left.-\frac{x_{4} K_{q p 2}+\left(C_{t p 2}+C_{e c 2}\right) x_{6}-A_{2} x_{8}}{A_{2}\left(L-l_{0}-x_{7}\right)}, 0,0\right)^{\mathrm{T}} \\
& \frac{\partial f}{\partial \alpha_{18}}=\left(0,0,0,0,0,0,0,-\frac{A_{1} x_{5}-A_{2} x_{6}-B_{p} x_{8}-K x_{7}-F_{L}}{m_{t}^{2}}\right)^{\mathrm{T}} \\
& \frac{\partial f}{\partial \alpha_{19}}=\left(0,0,0,0,0,0,0,-\frac{x_{8}}{m_{t}}\right)^{\mathrm{T}} \\
& \frac{\partial f}{\partial \alpha_{20}}=\left(0,0,0,0,0,0,0,-\frac{1}{m_{t}}\right)^{\mathrm{T}} \\
& \frac{\partial f}{\partial \alpha_{21}}=\left(0,0,0,0,0,0,0,-\frac{x_{7}}{m_{t}}\right)^{\mathrm{T}}
\end{aligned}
$$

上述求得的 $8 \times 21$ 阶矩阵为式(6)灵敏度函数的 自由项矩阵。 
由于液压缸的初始位置会影响其固有频率, 对 整个泵控系统控制性能产生影响, 为了探究初始液 压缸初始位置的影响程度, 将其设为一个单独的参 数 $\mathrm{l}_{0}$, 并认为主缸和回程缸的初始值为 0 , 故其状 态矢量 $\boldsymbol{x}$ 的初始值 $\boldsymbol{x}_{0}=\boldsymbol{0}$, 根据式(5)得灵敏度函数 $\lambda_{0}^{i}$ 的初始值为

$$
\lambda_{0}^{i}=0, \quad i=1,2, \cdots, 21
$$

\section{3 灵敏度函数计算}

灵敏度方程式(4)是带有时变系数项和时变自 由项的一阶线性非齐次微分方程组 $n \times(i+1)$ 。 $n$ 个状 态方程表达式以及 $n \times i$ 个灵敏度方程表达式, 这里 只需求活动横梁位移 $x_{7}$ 对参数矢量 $\boldsymbol{\alpha}$ 的灵敏度。

在 Matlab/Simulink 中针对活动横梁向下运动建 立开式洜控非对称缸系统的仿真模型, 并且得出调 整时间为 $0.2 \mathrm{~s}$, 其仿真模型参数及初始值如表 1 所示。

基于仿真模型, 利用变步长且计算精度较高的 四阶/五阶 Runge-Kutta 算法, 在 Matlab 中软件主界 面中进行编程, 可得出在外负载力作用下活动横梁 的输出位移 $\mathrm{x}_{7}$ 对参数 $\boldsymbol{\alpha}$ 的灵敏度函数时程曲线, 如 图 2 所示。同理, 可以求出其他工况下的灵敏度函 数时程曲线, 本文由篇幅所限, 不在此一一列举。

从图 2 中可以看出系统中各参数均在动态调整 时间内对活动横梁位移 $x_{7}$ 影响较大, 其中曲线正负
分别表示增大或抑制 $x_{7}$ 的变化, 而当 $x_{7}$ 趋于稳定值 附近时, $\alpha_{2} 、 \alpha_{4} 、 \alpha_{5} 、 \alpha_{6} 、 \alpha_{20} 、 \alpha_{21}$, 这些参数的灵 敏度函数不趋于 0 , 式(6)非齐次方程组有非零解, 即上述 6 个参数对系统稳态特性产生影响。

\section{表 1 仿真模型参数及初始值表}

\begin{tabular}{|c|c|}
\hline 仿真模型参数 & 初始值 \\
\hline 外负载力 $F_{L} / \mathrm{kN}$ & 10 \\
\hline 系统比例增益 $K_{P}$ & 5 \\
\hline 负载等效质量 $m_{t} / \mathrm{kg}$ & 10 \\
\hline 体积弹性模量 $\beta_{e} / \mathrm{Pa}$ & $8 \times 10^{8}$ \\
\hline 主缸的有效面积 $A_{1} / \mathrm{cm}^{2}$ & 78.6 \\
\hline 回程缸的有效面积 $A_{2} / \mathrm{cm}^{2}$ & 31.8 \\
\hline 负载弹簧刚度 $K /(\mathrm{N} / \mathrm{m})$ & 3300 \\
\hline 活动横梁初始位置 $l_{0} / \mathrm{mm}$ & 50 \\
\hline 粘性阻尼系数 $B_{p} /(\mathrm{N} /(\mathrm{m} / \mathrm{s}))$ & 5000 \\
\hline 主缸外泄漏系数 $C_{e c 1} /\left(\mathrm{m}^{3} /(\mathrm{s} \cdot \mathrm{Pa})\right)$ & $1.88 \times 10^{-13}$ \\
\hline 回程缸外泄漏系数 $C_{e c 2} /\left(\mathrm{m}^{3} /(\mathrm{s} \cdot \mathrm{Pa})\right)$ & $1.1 \times 10^{-13}$ \\
\hline 伺服变量䈋 2 外泄漏系数 $C_{t p 1} /\left(\mathrm{m}^{3} /(\mathrm{s} \cdot \mathrm{Pa})\right)$ & $3.1 \times 10^{-13}$ \\
\hline 伺服变量葲 1 外泄漏系数 $C_{t p 2} /\left(m^{3} /(s \cdot P a)\right)$ & $3.5 \times 10^{-13}$ \\
\hline 伺服变量葲 2 先导级伺服阀增益 $K_{\mathrm{s} 1} /\left(\mathrm{m}^{3} / \mathrm{s} \cdot \mathrm{A}\right)$ & 0.8 \\
\hline 伺服变量葲 1 先导级伺服阀增益 $K_{\mathrm{s} 2} /\left(\mathrm{m}^{3} / \mathrm{s} \cdot \mathrm{A}\right)$ & 0.2 \\
\hline 伺服变量葲 2 先导级伺服阀时间常数 $T_{s 1} / \mathrm{s}$ & 0.01 \\
\hline 伺服变量葲 1 先导级伺服阀时间常数 $T_{\mathrm{s} 2} / \mathrm{s}$ & 0.01 \\
\hline 伺服变量洜 2 先导级伺服阀油缸面积 $A_{s 1} / \mathrm{cm}^{2}$ & 28 \\
\hline 伺服变量洜 1 先导级伺服阀油缸面积 $A_{\mathrm{s} 2} / \mathrm{cm}^{2}$ & 20 \\
\hline 伺服变量泵 2 先导级伺服阀放大增益 $K_{\mathrm{a} 1} /(\mathrm{A} / \mathrm{V})$ & 0.12 \\
\hline 伺服变量洜 1 先导级伺服阀放大增益 $K_{\mathrm{a} 2} /(\mathrm{A} / \mathrm{V})$ & 0.12 \\
\hline
\end{tabular}

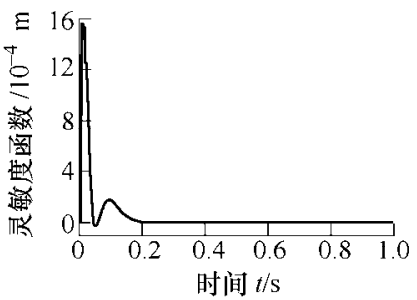

(a) 灵敏度函数 $\lambda \frac{1}{7}$

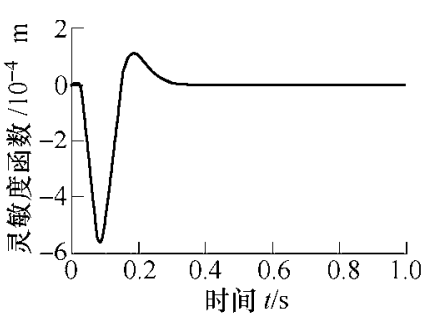

(d) 灵敏度函数 247

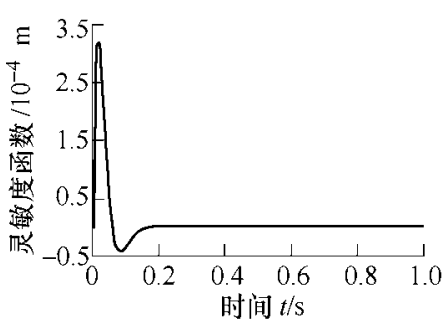

(g) 灵敏度函数 $\lambda_{7}^{7}$

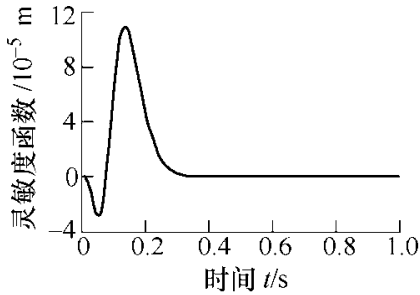

(b) 灵敏度函数 $\lambda_{7}^{2}$

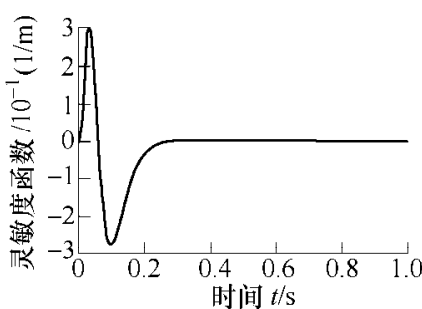

(e) 灵敏度函数 $\lambda_{7}^{5}$

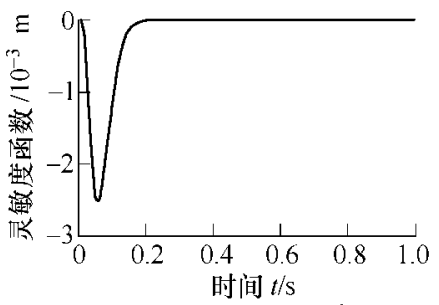

(h) 灵敏度函数 $\lambda \frac{8}{7}$

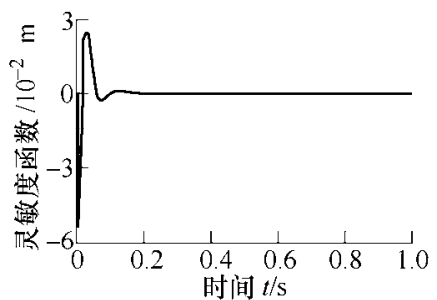

(c) 灵敏度函数 $\lambda \frac{3}{7}$

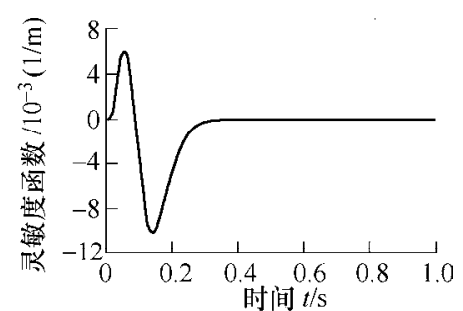

(f) 灵敏度函数 $\lambda_{7}^{6}$

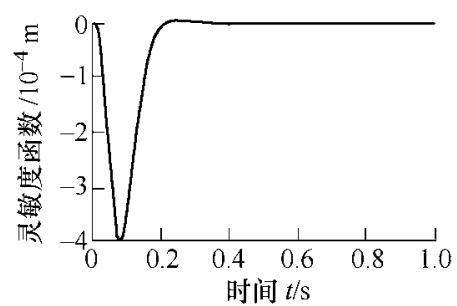

(i) 灵敏度函数 $\lambda \frac{9}{7}$ 


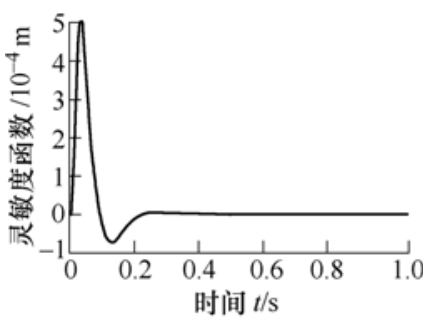

(j) 灵敏度函数 $\lambda_{7}^{10}$

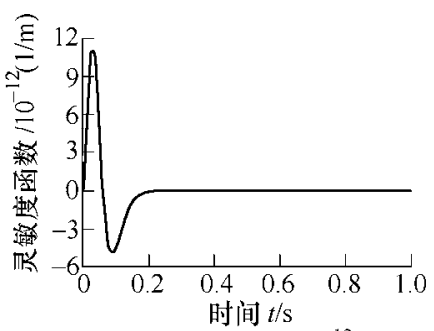

(m) 灵敏度函数 $\lambda^{13}$

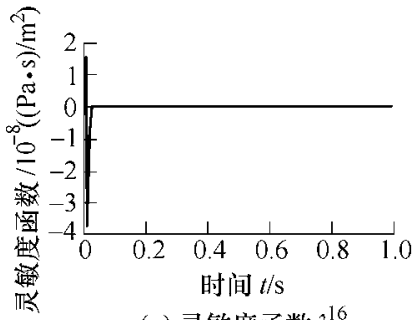

(p) 灵敏度函数 $\lambda_{7}^{16}$

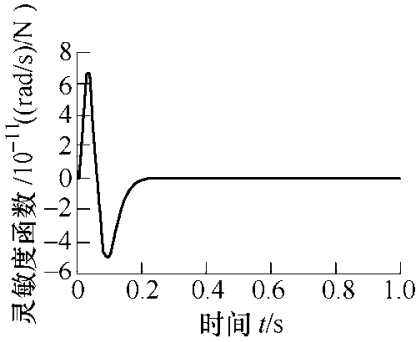

(s) 灵敏度函数 $\lambda_{7}^{19}$

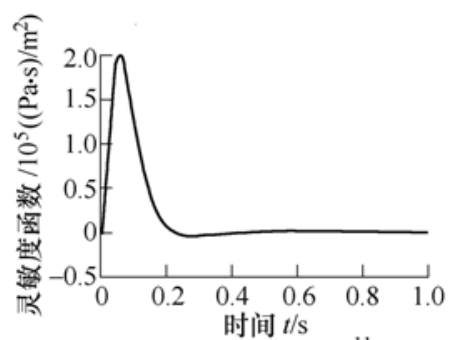

(k) 灵敏度函数 $\lambda_{7}^{11}$

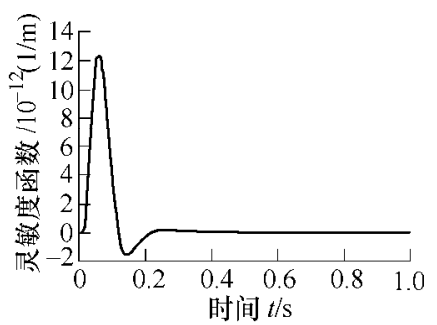

(n) 灵敏度函数 $\lambda_{7}^{14}$

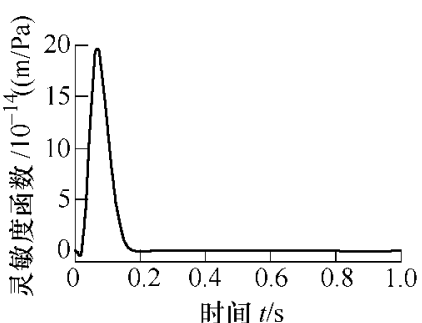

(q) 灵敏度函数 $\lambda_{7}^{17}$

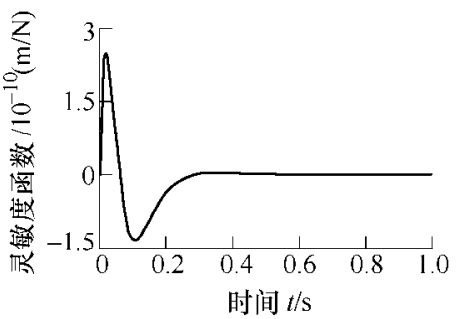

( $\mathrm{t}$ 录敏度函数 $\lambda_{7}^{20}$

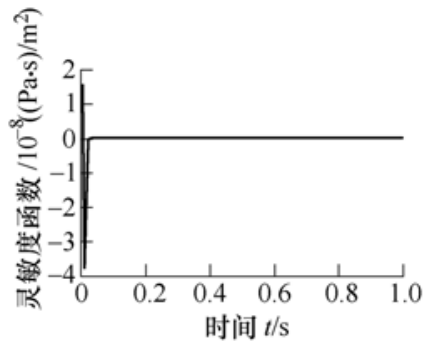

(1) 灵敏度函数 $\lambda 7_{7}^{12}$

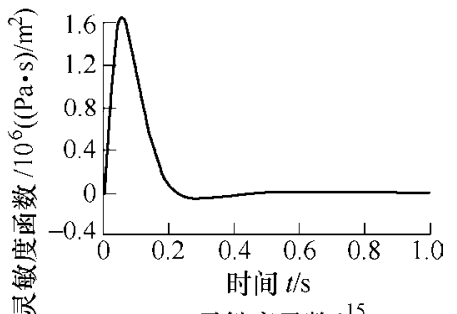

(o) 灵敏度函数 $\lambda_{7}^{15}$

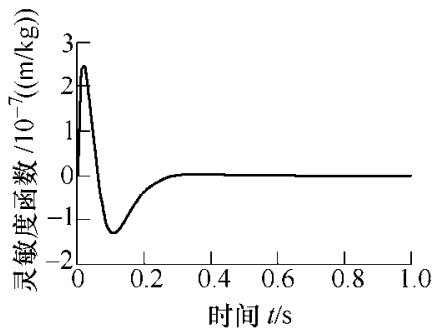

(r) 灵繁度函数 $\lambda_{7}^{18}$

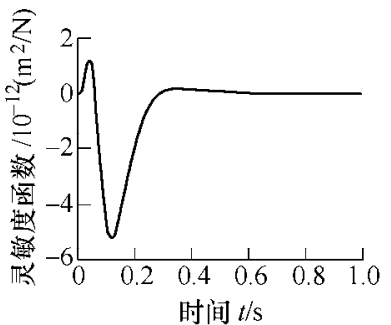

(u) 灵敏度函数 $\lambda_{7}^{2}$

图 2 开式百控系统 $F_{\mathrm{L}}=10000 \mathrm{~N}$ 活动横梁向下运动时程曲线

\section{3 开式洜控系统灵敏度分析}

\section{1 灵敏度指标}

图 2 中一阶轨迹灵敏度函数曲线描述了参数矢 量 $\boldsymbol{\alpha}$ 对开式泵控非对称缸系统位置阶跃影响的动态 变化过程, 为了更直观的量化参数矢量 $\boldsymbol{\alpha}$ 的影响程 度, 定义 2 种不同的灵敏度衡量指标。

矢量变化 $\Delta \boldsymbol{\alpha}$ 会引起状态矢量变化 $\Delta \boldsymbol{x}$, 代入式 (2)中求差, 并作泰勒展开, 可得

$$
\Delta \boldsymbol{x}=\left(\frac{\partial \boldsymbol{x}}{\partial \boldsymbol{\alpha}}\right)_{n} \Delta \boldsymbol{\alpha}+\text { 高阶项 }
$$

式中的 $(\partial \boldsymbol{x} / \partial \boldsymbol{\alpha})_{n}$ 即为一阶轨迹灵敏度函数 $\lambda_{n}^{i}$ 。

对于开式泵控非对称系统位移阶跃响应特性, 由于给定的位移和初始位移均为恒值, 因此采用参 数变化引起液压缸位移变化 $\Delta x_{7}$ 相对于其位移阶跃
量的稳态值 $x_{s j}$ 的百分比, 即相对于给定位移与初始 位移差值的百分比，来衡量各个参数变化对活塞杆 位移 $x_{7}$ 的影响程度。忽略式(10)中的高阶项, 把该 百分比的最大值作为第一项灵敏度衡量指标 $S_{1}$, 即 峰值灵敏度, 以下简称灵敏度指标 1 , 其表达式为

$$
s_{1}=\left.\frac{\Delta x_{j}}{x_{s j}}\right|_{\max } \times 100 \%=\left.\frac{\lambda_{j}^{i} \Delta \alpha_{i}}{x_{s j}}\right|_{\max } \times 100 \%
$$

为了衡量在采样时间内各参数变化对 $\Delta \mathrm{x}_{7}$ 影响 的总体程度, 将 $\left|\dot{\lambda}_{\alpha_{i}}\right| \cdot \Delta \alpha_{i}$ 在采样时间内对时间 $t$ 的 积分定义为均值灵敏度指标, 以下简称灵敏度指标 2 , 用 $S_{2}$ 表示, 其表达式为

$$
s_{2}=\int_{0}^{t_{0}}\left|\dot{\lambda}_{\alpha_{i}}\right| \cdot \Delta \alpha_{i} \mathrm{~d} t
$$

通过定义两种灵敏度衡量指标, 可定量分析各 参数矢量的变化 $\Delta \alpha$ 对开式泵控非对称缸系统位置 的影响程度。 


\section{2 开式洜控非对称缸系统灵敏度分析}

给定液压缸位置阶跃为 $50 \mathrm{~mm}$, 在空载和加载 工况且各参数变化 $10 \%$ 的情况下, 各参数矢量变化 $\Delta \alpha_{i}$ 引起液压缸位置变化的百分比时程曲线如图 3

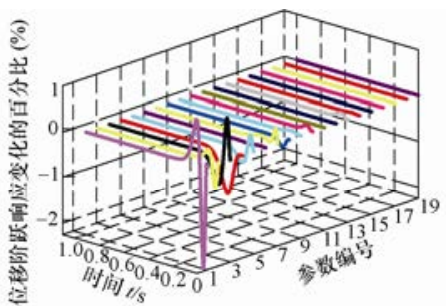

(a) 无负载工况下的时程曲线

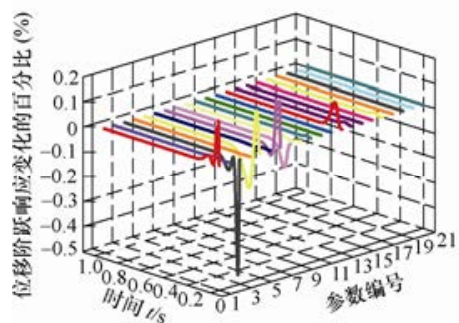

(c) $F_{\mathrm{L}}=30 \mathrm{kN}$ 时程曲线
所示。

根据灵敏度指标的定义，通过图 3 中各曲线得 到对应的灵敏度指标 1 和灵敏度指标 2 数值, 并用 柱形图表示，如图 4 所示。

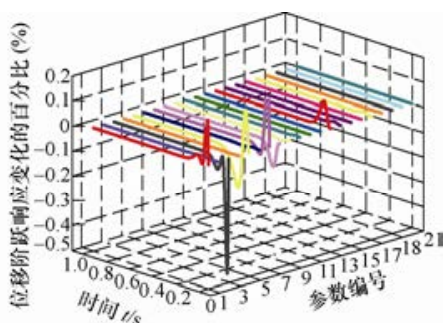

(b) $F_{\mathrm{L}}=10 \mathrm{kN}$ 时程曲线

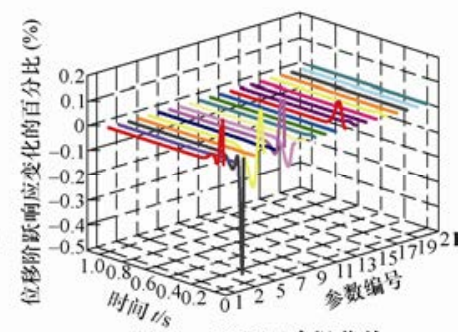

d) $F_{\mathrm{L}}=50 \mathrm{kN}$ 时程曲线

图 3 活动横梁向下运动各工况时程曲线

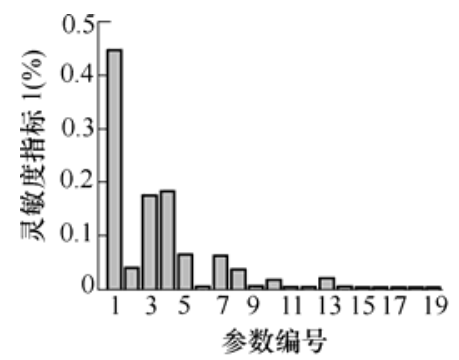

(a) 空载时灵敏度指标 1

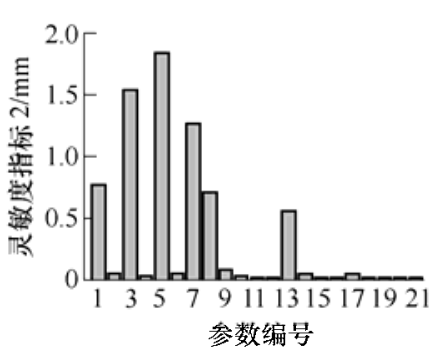

(d) $F_{\mathrm{L}}=10 \mathrm{kN}$ 时灵敏度指标 2

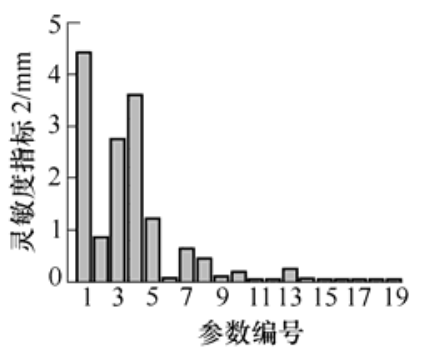

(b) 空载时灵敏度指标 2

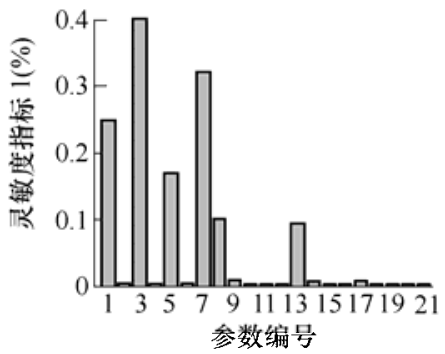

(e) $F_{\mathrm{L}}=30 \mathrm{kN}$ 时灵敏度指标 1

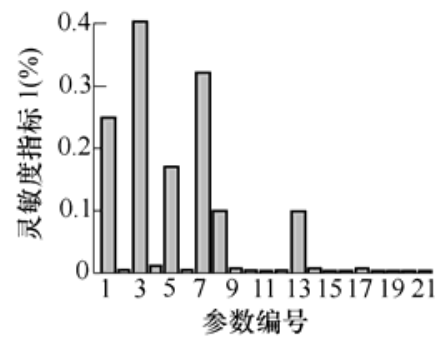

(c) $F_{\mathrm{L}}=10 \mathrm{kN}$ 时灵敏度指标 1

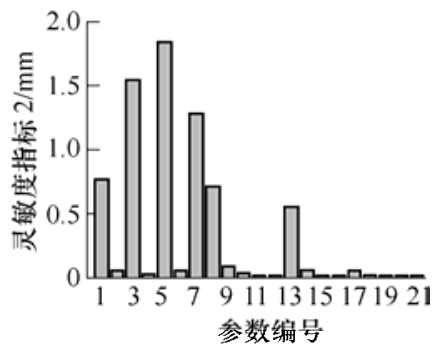

(f) $F_{\mathrm{L}}=30 \mathrm{kN}$ 时灵敏度指标 2

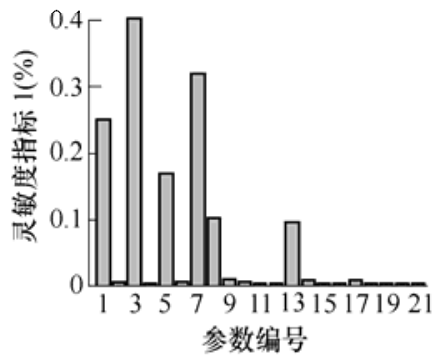

(g) $F_{\mathrm{L}}=50 \mathrm{kN}$ 时灵敏度指标

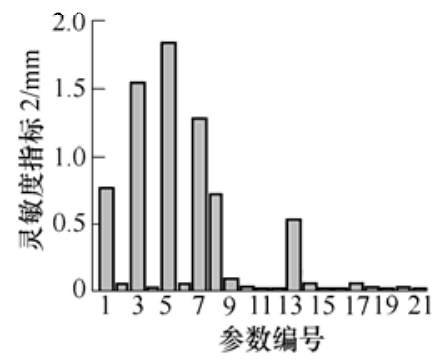

(h) $F_{\mathrm{L}}=50 \mathrm{kN}$ 时灵敏度指标 2

图 4 各参数灵敏度指标 
可以看出, 在不同工况下各参数的两项灵敏度 指标发生了不同程度的变化。在空载时, 伺服变量 泵 2 先导级伺服阀增益 $\alpha_{1}$ 、伺服变量葲 2 和伺服变 量洜 1 的先导级伺服阀时间常数 $\alpha_{3} 、 \alpha_{4}$ 的灵敏度指 标 1 均大于 $0.75 \%$ ，灵敏度指标 2 均超过 $2.5 \mathrm{~mm}$, 可知灵敏度指标 2 均超过 $7 \mathrm{~mm}$ 知这三个参数在动 态过程中对在动态过程中对 $x_{7}$ 影响较大。伺服变量 泵 1 先导级伺服阀增益和伺服变量洜 2 先导级油缸 面积 $\alpha_{2} 、 \alpha_{5}$, 比例增益 $\alpha_{7}$ 、伺服变量泵 2 流量增益 $\alpha_{8}$ 的灵敏度指标 1 均在 $0.25 \%$ 附近，灵敏度指标 2 趋势相同, 灵敏度指标 2 趋势相同, 影响程度次之, 其余参数的两项灵敏度指标很小, 影响程度较小。

在不同外负载力作用下，各参数两项灵敏度指 标数值相近, 其中 $\alpha_{1} 、 \alpha_{3} 、 \alpha_{5} 、 \alpha_{7}$ 和 $\alpha_{8}$ 的灵敏度指 标 1 均大于 $0.1 \%$, 且灵敏度指标 2 均大于 $0.5 \mathrm{~mm}$, 可知灵敏度指标 2 均超过 $7 \mathrm{~mm}$ 知这五个参数在动 态过程中对在动态过程中对 $x_{7}$ 影响较大, 且灵敏度 指标 2 均大于 $1 \mathrm{~mm}$, 可知这六个参数在动态过程中 对活动横梁位移 $x_{7}$ 影响较大。其余参数的变化对 $x_{7}$ 影响较小。与空载相比, 先导级伺服阀时间常数 $\alpha_{3}$, 伺服变量泵 2 流量增益 $\alpha_{8}$ 与系统比例增益 $\alpha_{7}$ 两项灵 敏度指标在柱形图中相对于纵坐标轴所占比例(以 下简称: 所占比例)增大两项灵敏度指标在柱形图中 相对于纵坐标轴所占比例(以下简称：所占比例)增 大。伺服变量洜 1 先导级伺服阀时间常数 $\alpha_{4}$ 两项灵 敏度指标数值很小, 对 $x_{7}$ 影响程度较空载大幅减小。 主缸有效面积 $\alpha_{13}$ 两项灵敏度指标所占比例增大, 但数值与空载时相近。

\section{4 开式洜控非对称缸系统灵敏度试验}

\subsection{6 MN 开式洜控油压机试验平台}

$0.6 \mathrm{MN}$ 开式泵控锻造油压机, 液压系统主要包 括 MOOG 三联洜组、电动机、原头阀组、低压补油 系统和检测元件等, $0.6 \mathrm{MN}$ 开式洜控油压机试验平 台如图 5 所示, 液压系统参数如表 2 所示。

表 $20.6 \mathrm{MN}$ 洜控油压机液压系统参数

\begin{tabular}{lc}
\hline \multicolumn{1}{c}{ 名称 } & 参数 \\
\hline 公称压力 $/ \mathrm{kN}$ & 550 \\
回程力 $/ \mathrm{kN}$ & 55 \\
最大行程 / m & 0.12 \\
工进最高速度 / (m/s) & 0.05 \\
空程快下最高速度 / (m/s) & 0.06 \\
回程最高速度 / (m/s) & 0.06 \\
主缸内径 / $\mathrm{mm}$ & 100 \\
回程缸内径 / mm & 45 \\
\hline
\end{tabular}

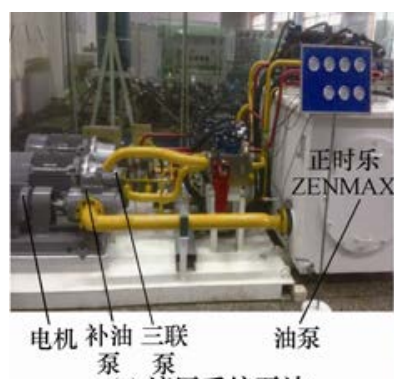

(a) 液压系统泵站

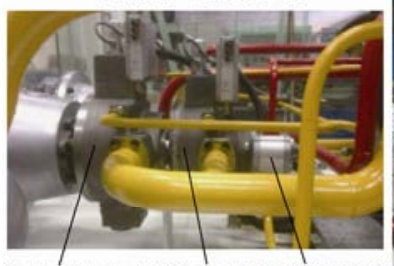

主缸变量泵 回程缸变量㫳 控制油泵

(b) MOOG 三联原

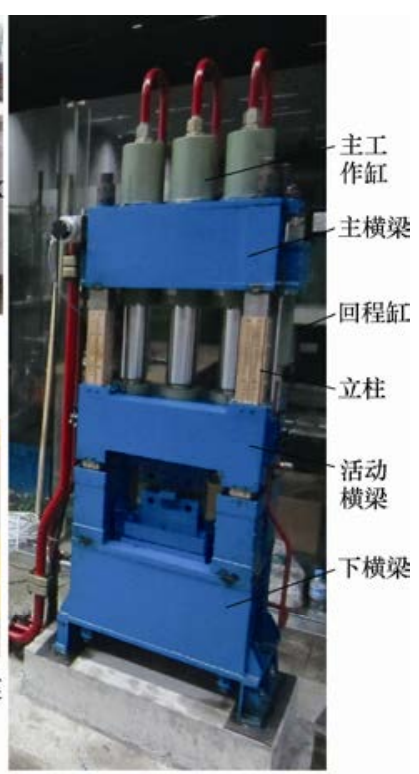

(c) $0.6 \mathrm{MN}$ 液压机
图 $50.6 \mathrm{MN}$ 开式葲控油压机试验台

试验台的数据采集控制系统要包括压力、位 移传感器和 xPC-Target 控制器等。其中运动横梁 位移采用两台由拉线盒和旋转编码器组合成的拉 线式位移传感器进行采集, 采集的数据通过由 LABVIEW 图形化开发工具搭建的控制采集界面 得以观测和保存。

\section{2 开式洜控系统灵敏度指标试验验证}

由于开式䈋控系统可控参数较少, 本文采用类 比法, 只验证部分参数的灵敏度分析结果, 因为系 统的比例增益 $\alpha_{7}$ 和活动横梁的初始位置 $\alpha_{10}$ 容易控 制, 因此, 对以上两个参数在试验负载力为 $30 \mathrm{kN}$, 活动横梁阶跃响应为 $50 \mathrm{~mm}$ 条件下的两项灵敏度指 标进行验证，测试曲线如图 6 所示。
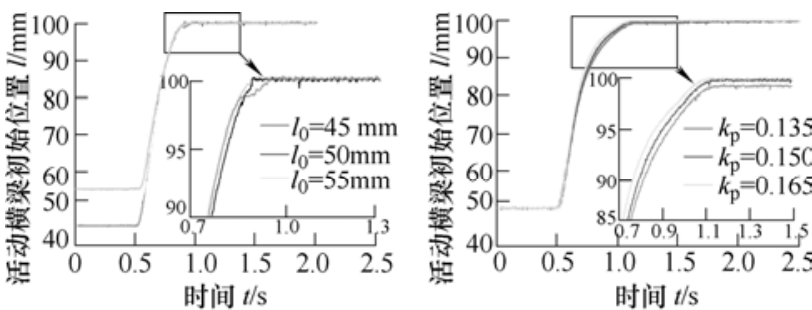

(a) 初始位置改变 $10 \%$ 测试曲线 (b) 比例增益改变 $10 \%$ 测试曲线 图 $6 F_{\mathrm{L}}=30 \mathrm{kN}$ 时系统测试曲线图

由图 6 可知, 对活动横梁初始位置改变 10\%时 对系统动静态特性影响较小, 而系统比例增益改变 $10 \%$ 时, 对系统动态特性影响较大, 且调整时间随 着系统比例增益的增大而减小，这与上述灵敏度分 析结果一致，初步证实了理论分析的准确性。

将测得主缸的输出位移与参数变化前的输出位 移相减, 求出偏差的最大值以及各偏差的绝对值之 和, 为了保证试验结果的准确性, 采用多样本求均 
值的方法, 整理得出上述 2 个参数的两项灵敏度指 标试验值与仿真对比柱形图，如图 7、8 所示。

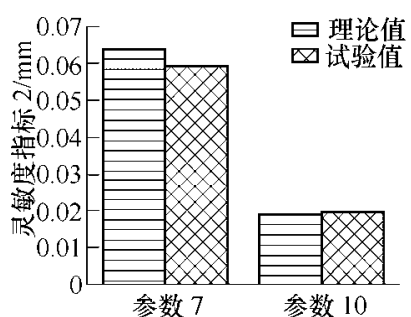

(a) 灵敏度指标 1

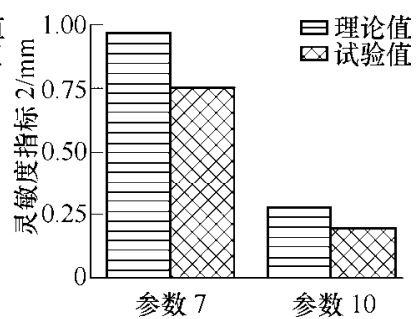

(b) 灵敏度指标 2
图 7 空载工况下部分参数灵敏度指标对比图

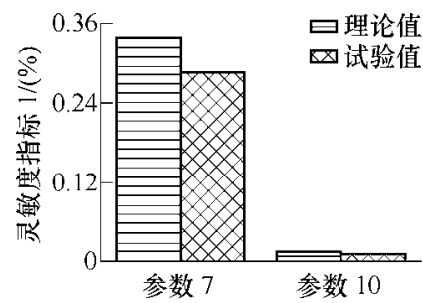

(a) 灵敏度指标

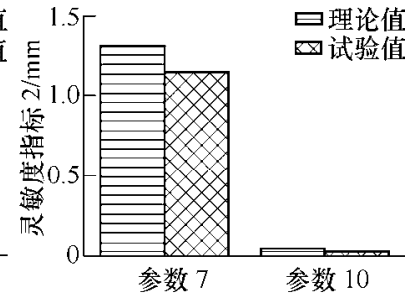

(b) 灵敏度指标 2
图 8 加载工况下部分参数灵敏度指标对比图

通过图 7、8 可以看出, 两种工况下以上两个参 数的灵敏度指标实验值与理论值相差不大, 加载工 况下灵敏度指标理论值大于试验值, 这是负载力存 在一定的波动。但是每个参数的灵敏度指标的所占 比例与理论是相吻合的, 这充分的证实了理论分析 的准确性。

\section{5 结论}

(1) 建立了开式洜控非对称缸系统数学模型, 应用灵敏度分析理论, 在一定的初始条件下, 研究 了在开式洜控系统中各参数变化 $10 \%$, 对其位移阶 跃响应性能的影响, 通过 $0.6 \mathrm{MN}$ 开式泵控油压机 试验台, 采用多样本求均值的方法对理论分析结果 进行验证。

（2）伺服变量洜 2 的先导级伺服阀的增益 $\alpha_{1}$ 、 时间常数 $\alpha_{3}$ 和油缸面积 $\alpha_{5}$ 以及流量增益 $\alpha_{8}$ 小范围 波动, 在动态过程中对活塞杆位移在动态运动中对 $x_{7}$ 有较大的影响, 因此需要洜具有良好的性能, 在 设计补偿控制策略时应重点考虑以上参数的摄动。

(3) 主缸的有效面积 $\alpha_{13}$ 在两种工况下两项灵敏 度指标数值相近, 但在负载存在时灵敏度指标所占 比例较大, 因此在对主缸设计时, 应根据系统要求 进行寻优设计。

（4）伺服变量泵 1 的先导级伺服阀的增益 $\alpha_{2}$ 、 时间常数 $\alpha_{4}$ 、油缸面积 $\alpha_{6}$, 伺服变量泵 2 的先导级 伺服阀油缸面积 $\alpha_{5}$ 、外负载力 $\alpha_{20}$ 、粘性阻尼系数 $\alpha_{21}$, 会对 $x_{7}$ 的稳态特性产生不同程度影响, 其余参
数则影响较小。

\section{参 考 文 献}

[1] 权龙. 原控缸电液技术研究现状、存在问题及创新解决 方案[J]. 机械工程学报, 2008，44(11): 87-92.

QUAN Long. Current state, problems and the innovative solution of electro-hydraulic technology of pump controlled cylinder[J]. Chinese Journal of Mechanical Engineering, 2008, 44(11): 87-92.

[2] MINAV T A, LAURILA L I E, PYRHUENRN J J. Analysis of electro-hydraulic lifting system's energy efficiency with direct electric drive pump control[J]. Automation in Construction, 2013, 30: 144-150.

[3] QUAN Zhongyi, QUAN Long, ZHANG Jinman. Review of energy efficient direct pump controlled cylinder electro-hydraulic technology[J]. Renewable and Sustainable Energy Reviews， 2014， 35: 336-346.

[4] 徐绳武. 百控系统在国外的发展 $[\mathrm{J}]$. 液压气动与密封, 2010, 30(3): 1-4.

XU Shengwu. Development of pump-controlled system abroad[J]. Hydraulics Pneumatics and Seals, 2010, 30(3): $1-4$.

[5] 景健, 权龙, 黄家海, 等. 非对称泵直驱液压挖掘机斗 杆特性研究[J]. 机械工程学报, 2016, 52(6): 188-196. JING Jian, QUAN Long, HANG Jiahai, et al. Research on the characteristics of asymmetric pump directed controlled arm cylinder of excavator[J]. Journal of Mechanical Engineering, 2016, 52(6): 188-196.

[6] UWE K. A hydraulic high-speed tryout press for the simulation of mechanical forming processes[J]. Journal of Materials Processing Technology, 2001,111(1): 159-163.

[7] 姜继海, 苏文海, 张洪波, 等. 直驱式容积控制电液伺 服系统及其在船舶舵机上的应用 [J]. 中国造船, 2004(4): 58-63.

JIANG Jihai, SU Wenhai, ZHANG Hongbo, et al. Direct drive variable speed electro-hydraulic servo system to position control of a ship Rudder[J]. Shipbuilding of China, 2004(4): 58-63.

[8] TSAO T C, TOMIZUKA M. Robust adaptive and repetitive digital tracking control and application to a hydraulic servo for noncircular machining[J]. Journal of Dynamic Systems, Measurement and Control Transactions of the ASME, 1994, 116(1): 24-32.

[9] TO K, YAMADA T, IKEO S, et al. Application of simple adaptive control to water hydraulic servo cylinder system[J]. Chinese Journal of Mechanical Engineering, 2012, 25(5): 882-888.

[10] LIU R, ALLEYNE A. Nonlinear force/pressure tracking of an electro-hydraulic actuator[J]. Journal of Dynamic 
Systems, Measurement and Control Transactions of the ASME, 2000, 122(1): 232-237.

[11] GUAN Cheng, PAN Shuangxia. Adaptive sliding mode control of electro-hydraulic system with nonlinear unkNown parameters[J]. Control Engineering Practice, 2008, 16(11): 1275-1284.

[12] CHO S H, BURTON R. Position control of high performance hydrostatic actuation system using a simple adaptive control (SAC) method[J]. Mechatronics, 2010, 21(1): 109-115.

[13] KYOUNG K A, QUANG T D. Self-tuning of quantitative feedback theory for force control of an electro-hydraulic test machine[J]. Control Engineering Practice, 2009, 17(11): 1291-1306.

[14] SONG X M, KONG F Z, ZHAN C S, et al. Parameter identification and global sensitivity analysis of Xin’anjiang model using meta-modeling approach[J]. Water Science and Engineering, 2013， 6(1): 1-17.

[15] HALL J W, BOYCE S A, WANG Y L, et al. Sensitivity analysis for hydraulic models[J]. Journal of Hydraulic Engineering, 2009, 135(11): 959-969.

[16] WEI Pengfei, SONG Jingwen, LU Zhenzhou. Global reliability sensitivity analysis of motion mechanisms[J]. Journal of Risk and Reliability， 2016， 230： 265-277.

[17] 王新刚, 张义民, 王宝艳. 机械零部件的动态可靠性灵 敏度分析[J]. 机械工程学报, 2010, 46(10): 188-193. WANG Xingang, ZHANG Yimin, WANG Baoyan. Dynamic reliability sensitivity analysis of mechanical components[J]. Journal of Mechanical Engineering, 2010, 46(10): 188-193.

[18] VERHOEVEN R. Analysis of the workspace of tendon-based Stewart platform[D]. Duisburg, Germany: Gerhard Mercator University, 2004.
[19] BA Kaixian, YU Bin, KONG Xiangdong, et al. The dynamic compliance and its compensation control research of the highly integrated valve-controlled cylinder position control system[J]. International Journal of Control, Automation \& SystemsJournal of Dynamic Systems, 2017, 15(4): 1814-1825.

[20] BA Kaixian, YU Bin. Parameters sensitivity analysis of position-based impedance control for bionic legged robots' HDU[J]. Applied Sciences， 2017， 7(10): 1035.

[21] ANTON I, LOAN S. Numerical modeling of cavitation characteristics and sensitivity curves for reversible hydraulic machinery[J]. Engineering Analysis with Boundary Elements, 2014, 41: 18-27.

[22] 姚静, 任旭辉, 曹晓明, 等. 开式变量泵控快锻油压机 系统能耗特性实验研究[J]. 中国机械工程, 2017, 28(4): 462-470.

YAO Jing, REN Xuhui, CAO Xiaoming, et al. Experimental study on energy consumption characteristics of fast hydraulic forging press with open variable pump-controlled system[J]. China Mechanical Engineering, 2017, 28(4): 462-470.

[23] 姚静, 王佩, 董兆胜, 等. 开式洜控非对称缸负载容腔 独立控制耦合特性[J]. 中国机械工程，2017，28(14): 1639-1645.

YAO Jing, WANG Pei, DONG Zhaosheng, et al. Coupling characteristics of independent volume-in and volume-out control for open circuit pump-controlled asymmetric cylinder systems $[\mathrm{J}]$. Chian Mechanical Engineering, 2017, 28(14): 1639-1645.

作者简介: 姚静(通信作者), 女, 1978 年出生, 博士研究生。主要研究 方向为重型机械流体传动与控制系统和新型液压元件。

E-mail: jyao@ysu.edu.cn 